\title{
A New Control Method for Three Phase Inverters under Unsymmetrical Voltage Sag Conditions
}

\author{
Jiabin Shi ${ }^{1}$, Liang $\mathrm{Ji}^{1}$, Qiteng Hong ${ }^{2}$, Yang $\mathrm{Mi}^{1}$, Zhe $\mathrm{Cao}^{1}$, Asif $\mathrm{Khan}^{2}$, Campbell Booth ${ }^{2}$ \\ 1. Shanghai University of Electric Power, 200090, Shanghai, China \\ 2. University of Strathclyde, G1 1RD, Glasgow, UK \\ Corresponding author: Liang Ji, E-Mail: liangji@ shiep.edu.cn
}

\begin{abstract}
Under grid fault conditions, especially the unbalanced grid faults, the PCC voltage of DG will suffer notably unbalanced voltage droops, which may cause the unnecessary disconnection of DGs according to the grid codes. Moreover, the overcurrent risk during voltage sag will also result in the disconnection of DGs, and even damage the inverter. In this paper, a new fault control strategy including three control objectives, was proposed to enhance the low-voltage ride-through (LVRT) capability for three-phase inverters. Firstly, the positive sequence (PS) voltage method is proposed to maximize the voltage support capability in any types of unbalanced voltage sags. As to ensure the safe operation of the inverter, a current limitation algorithm is designed based on different operation scenarios. Also, the active power delivery is considered as an ancillary service to fully use the capacity of the inverter. Then, a new control method towards the scenario classification and reference current selection is proposed to simultaneously achieve these control objectives. Finally, the simulation results based on MATLAB/Simulink are presented to verify the effectiveness of the proposed fault control strategy.
\end{abstract}

Index Terms-- fault control strategy, grid faults, voltage sag , inverter interfaced distributed generation (IIDG), positive sequence (PS) voltage support, current limitation

\section{INTRODUCTION}

In the last decade, the requirement to promote the rapid development of renewable energy resources (RER) has become strategy goals of many countries due to the increasing environmental issues and energy crisis [1]. Distributed renewable energy (e.g. solar and wind energy) are regarded as effective ways to solve these problems. However, these distributed generations (DGs) are facing with growing challenges in guaranteeing the safe and continuate operation of them during various perturbations in power system, such as grid faults or load fluctuation [2]. And this challenge will be even greater if the DG is not properly controlled during the unbalanced perturbations like unbalanced grid faults.

In order to enhance the efficient operation of DG and maintain the reliability of the power system under unbalanced voltage conditions, voltage support requirements are involved in the recent grid codes [3]. In addition, the safe operation of DG also needs to be considered, which includes avoiding the overvoltage and overcurrent risks.

Recently, various control strategies with different control objectives have been investigated for inverter interfaced DG (IIDG). Some researches focus on the flexible control of output power and ensure the current limitation, [4-9]. Symmetrical components-based fault control strategies are presented in [4-6] to regulate the active and reactive power oscillations. However, the voltage support ability and current limitation are not fully emphasized. As to properly control the output current of the inverter, different control methods are investigated, where balanced current control is considered in [7] for IIDG and different current limiting algorithms are applied in [8-9]. In terms of voltage support, recent researches mainly focusing on the positive and negative sequence (NS) voltage control [10-11], which aims to regulate the voltage within the required ranges. However, large inverter capacity is needed to ensure such control objective.

Previous researches have investigated various control objectives for IIDGs during the unbalanced voltage sags. However, most of them only involve two or less control objectives. Therefore, this paper presents a fault control strategy which contains three control objectives to fully enhance the ride-through capability of the IIDG. Owing to the PS voltage control method, the maximization of the voltage support ability is guaranteed. Meanwhile, the phase current limitation algorithm is designed upon the three classified operation scenarios. To fully use the inverter capacity, both active current and NS current are regarded as ancillary services.

\section{IIDG PERFORMANCE DURING UnBALANCED VOLTAGE SAG CONDITION}

This section describes the operation of the IIDG during unbalanced voltage condition, which are necessary for the design of the controller. Fig. 1 shows the simplified circuit diagram used in this research, involving an IIDG connected to the main grid via a conductor whose impedance is mainly 
inductive. During the unbalanced voltage condition, the voltage vector can be expressed in the $\alpha \beta$ frame as

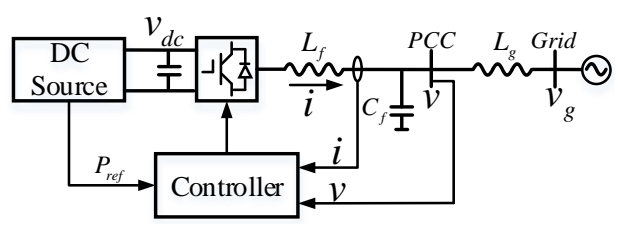

Figure 1. Simplified circuit diagram of an IIDG system

$$
v_{\alpha \beta}=\left[\begin{array}{c}
v_{\alpha}^{+}+v_{\alpha}^{-} \\
v_{\beta}^{+}+v_{\beta}^{-}
\end{array}\right]=\left[\begin{array}{c}
V^{+} \cos \left(\omega t+\varphi^{+}\right)+V^{-} \cos \left(-\omega t+\varphi^{-}\right) \\
V^{+} \sin \left(\omega t+\varphi^{+}\right)+V^{-} \sin \left(-\omega t+\varphi^{-}\right)
\end{array}\right]
$$

where $v_{\alpha}^{+}, v_{\beta}^{+}, v_{\alpha}^{-}$and $v_{\beta}^{-}$denote the PS and NS components of the voltage, $V^{+}$and $V^{-}$represent the magnitudes of the PS and NS sequence voltage, and $\varphi^{+}$and $\varphi^{-}$represent the initial phase angle of them. The instantaneous $\alpha \beta$ components of the current vector $i$ can be obtained as

$$
\begin{aligned}
i=i^{+}+i^{-}=\left[\begin{array}{c}
i_{\alpha} \\
i_{\beta}
\end{array}\right] & =\left[\begin{array}{c}
I_{p}^{+} \cos \left(\omega t+\varphi^{+}\right)-I_{q}^{+} \sin \left(\omega t+\varphi^{+}\right) \\
I_{p}^{+} \sin \left(\omega t+\varphi^{+}\right)+I_{q}^{+} \cos \left(\omega t+\varphi^{+}\right)
\end{array}\right] \\
& {\left[\begin{array}{c}
I_{p}^{-} \cos \left(-\omega t+\varphi^{-}\right)-I_{q}^{-} \sin \left(-\omega t+\varphi^{-}\right) \\
I_{p}^{-} \sin \left(-\omega t+\varphi^{-}\right)+I_{q}^{-} \cos \left(-\omega t+\varphi^{-}\right)
\end{array}\right] }
\end{aligned}
$$

where $I_{p}^{+}$and $I_{q}^{+}$are the PS active and reactive current amplitudes while $I_{p}^{-}$and $I_{q}^{-}$are the NS active and reactive current amplitudes. Due to the PS voltage support objective utilized in this paper, the PS voltages at PCC is obtained as

$$
v^{+}=v_{g}^{+}+L_{g} \frac{d i^{+}}{d t}
$$

where $v^{+}$and $v_{g}^{+}$denote the PS voltage at PCC and grid side bus respectively, and $L_{g}$ denotes the grid inductance. The magnitude of $v_{g}^{+}$can be obtained by inserting (2) to (3) as

$$
V_{g}^{+}=\sqrt{\left(V^{+}-\omega L_{g} I_{q}^{+}\right)^{2}+\left(\omega L_{g} I_{p}^{+}\right)^{2}}
$$

\section{CONTROl OBJeCtives UNDER Unbalanced Voltage CONDITIONS}

In order to improve the performance of the IIDG during unbalanced voltage conditions, a new control method which considers three objectives is proposed in this section.

\section{A. Objective 1: Active Power Control}

This part aims to achieve the active power control by injecting the required PS active current, which defined as the initial active current reference. This value may be modified upon the operation constraints such as phase voltage and phase current limitations. During unbalanced voltage conditions, the active power can be written as

$$
P=V^{+} I_{p}^{+}+V^{-} I_{p}^{-}
$$

By injecting the proper PS and NS active current, the required active power can be delivered to the grid. In the proposed control strategy, the reference current $I_{p}^{-}$is setting as zero, which means the active power is only injected via $I_{p}^{+}$. Thus, the initial reference current for $I_{p}^{+}$can be obtained as

$$
I_{p_{-} i n i}^{+}=\frac{P_{r e f}}{V^{+}}
$$

where $P_{\text {ref }}$ represents the active power reference.

\section{B. Objective 2: PS Voltage Maximization}

Under voltage sag conditions, voltage support is regarded as an important factor to assess the LVRT capability. Meanwhile, the three phase voltages should be well controlled without overvoltage risk. To ensure this, the following phase voltage constraints must be considered in the controller

$$
V_{\max }=\max \left\{V_{a}, V_{b}, V_{c}\right\} \leq V_{\text {upper }}
$$

where $V_{\max }$ notes the maximum phase voltage amplitude at PCC, and $V_{\text {upper }}$ notes threshold for the upper voltage limits, which is setting as 1.1 p.u.. The amplitude of the maximum phase voltage at PCC can be expressed as a function of the phase angle and the sequence components of the voltage

$$
V_{\max }^{2}=\left(V^{+}\right)^{2}+\left(V^{-}\right)^{2}+2 V^{+} V^{-} \lambda_{\max }
$$

where

$$
\lambda_{\max }=\max \left\{\cos (2 \gamma), \cos \left(2 \gamma+\frac{2 \pi}{3}\right), \cos \left(2 \gamma-\frac{2 \pi}{3}\right)\right\}
$$

and $\gamma=\frac{\varphi^{+}+\varphi^{+}}{2}$ represents the angle between the $\alpha$ axis and the long axis of the voltage vector locus. By inserting (7) to (8), the PS voltage reference can be derived as

$$
V_{r e f}^{+}=-V^{-} \lambda_{\text {max }}+\sqrt{\left(V^{-} \lambda_{\text {max }}\right)^{2}-\left[\left(V^{-}\right)^{2}-\left(V_{\text {upper }}\right)^{2}\right]}
$$

By solving (4) and (10), the PS reactive current $I_{q}^{+}$can be obtained to achieve the initial voltage support requirement

$$
I_{q_{-} i n i}^{+}=\frac{V_{r e f}^{+}-\sqrt{\left(V_{g}^{+}\right)^{2}-\left(X_{g} I_{p}^{+}\right)^{2}}}{X_{g}}
$$

\section{Objective 3: Phase Current Limitation}

This section aims to derive the current references which guarantee the phase currents to be well controlled within the 
limitations. Firstly, the magnitude of the three phase currents are derived by solving (2) as

$$
I_{a b c}=\sqrt{\left(A_{1}\right)^{2}+\left(A_{2}\right)^{2}}
$$

where

$$
\begin{aligned}
& A_{1}=\left(I_{p}^{+}+I_{p}^{-}\right) \cos \delta+\left(I_{p}^{+}-I_{p}^{-}\right) \sin \delta \\
& A_{2}=-\left(I_{q}^{+}-I_{q}^{-}\right) \cos \delta+\left(I_{q}^{+}+I_{q}^{-}\right) \sin \delta
\end{aligned}
$$

and $I_{a b c}$ denotes the magnitude of the three phase currents, which are obtained by choosing three different values of $\delta$ $\left(\delta=\gamma, \gamma+\frac{\pi}{3}, \gamma-\frac{\pi}{3}\right)$. Note that the proposed current limiting algorithm is highly depending on the current injection modes, which determined by the operation scenarios (e.g. severity of the sag and the level of the initial active power). And the scenarios are depending on the initial reference currents $\left(I_{p_{-} i n i}^{+}\right.$ and $I_{q_{-} i n i}^{+}$), which will be discussed in section IV.

\section{1) Mode 1: only $I_{q}^{+}$is injected to the grid}

This mode usually happens during severe voltage sags scenario, which leads large calculated initial reference current $I_{q_{-} i n i}^{+}$. However, the injection of this current may result in overcurrent risk $\left(I_{q_{-} i n i}^{+}>I_{\max }\right)$. Therefore, as to ensure the phase current limitation objective, $I_{q_{-} i n i}^{+}$should be modified as

$$
I_{q_{-} \text {ref }}^{+}=I_{\max }
$$

where $I_{\max }$ notes the maximum allowable output current.

2) Mode 2: both $I_{p}^{+}$and $I_{q}^{+}$are injected to the grid

For unserious voltage sag and high active power generation scenario, the initial reference current $I_{q_{-} i n i}^{+}$is lower than $I_{\text {max }}$. However, the combined injection of the initial active current $I_{p_{-} i n i}^{+}$causes the overcurrent risk. Therefore, considering the that both $I_{p}^{+}$and $I_{q}^{+}$are injected in this mode, $I_{p_{-} i n i}^{+}$should be recalculated based on the restriction of the phase current as

$$
I_{p_{-} \text {ref }}^{+}=\sqrt{\left(I_{\max }\right)^{2}-\left(I_{q_{-} i n i}^{+}\right)^{2}}
$$

\section{3) Mode 3: $I_{p}^{+}, I_{q}^{+}$and $I_{q}^{-}$are injected to the grid}

For unserious voltage sag and low active power generation scenario, both initial control objectives 1 and 2 are satisfied by injecting $I_{p_{-} i n i}^{+}$and $I_{q_{-} i n i}^{+}$, and the currents are well controlled below $I_{\max }$. As to fully use the inverter capacity, the NS reactive current $I_{q}^{-}$is required to be injected, which can decrease the NS voltage. Based on the initial reference current
$I_{p_{-} i n i}^{+}$and $I_{q_{-} i n i}^{+}$, and setting $I_{p_{-} r e f}^{-}$as zero, the reference current for $I_{q}^{-}$is derived by solving (12) and (13), which is

$$
I_{q_{-} a b c}^{-}=A+\sqrt{(A)^{2}-B}
$$

where

$$
\left\{\begin{array}{l}
A=I_{q_{-} i n i}^{+} \cos (2 \delta)-I_{p_{-} i n i}^{+} \sin (2 \delta) \\
B=\left(I_{p_{-} i n i}^{+}\right)^{2}+\left(I_{q_{-} i n i}^{+}\right)^{2}-\left(I_{\max }\right)^{2}
\end{array}\right.
$$

and $I_{q_{-} a b c}^{-}$denotes the reactive current references when the currents in phase a, b and c reach to $I_{\max }$ respectively, which is obtained by inserting different $\delta$ into (16) and (17). To avoid the overcurrent risk, the final reference current is choosing as the minimum value of $I_{q_{-} a b c}^{-}$, which is written as

$$
I_{q_{-} r e f}^{-}=\min \left(I_{q_{-} a}^{-}, I_{q_{-} b}^{-}, I_{q_{-} c}^{-}\right)
$$

where $I_{q_{-} a}^{-}, I_{q_{-} b}^{-}$and $I_{q_{-} c}^{-}$are the reference current components of $I_{q_{-} a b c}^{-}$.

\section{Proposed Control Method Under Unbalanced VolTAge SAg CONDITIONS}

In this section, the classification principle of the operation scenario and the determination of the current injection mode are discussed in detail, which have been implemented to achieve objective 3 in section C. Fig. 2 shows the control block of the proposed reference current calculation algorithm.

\section{1) Operation scenario classification}

The initial reference currents $I_{p_{-} i n i}^{+}$and $I_{q_{-} i n i}^{+}$derived by(6) and (11)are serve to classify the operation scenarios based on the depth of the sag and the level of the active power .

For $I_{q_{-} \text {ini }}^{+}>I_{\max }$, the scenario is defined as serious voltage sag and, for $I_{q_{-} i n i}^{+} \leq I_{\max }$, the scenario is defined as unserious voltage sag. To evaluate the level of the active power generation, the initial PS current $I_{i n i}^{+}$is defined as follow:

$$
I_{i n i}^{+}=\sqrt{\left(I_{q_{-} i n i}^{+}\right)^{2}+\left(I_{p_{-} i n i}^{+}\right)^{2}}
$$

If the combined injection of $I_{p_{-} i n i}^{+}$causes the overcurrent risk $\left(I_{q_{-} \text {ini }}^{+} \leq I_{\text {max }}\right.$, but $\left.I_{\text {ini }}^{+}>I_{\text {max }}\right)$, the scenario is defined as high level active power generation, and for case of $I_{i n i}^{+}<I_{\text {max }}$, the scenario matches with low active power generation.

\section{1) Reference Current Determination}

In the proposed control method, three operation scenarios are considered to determine the final reference current. Each 


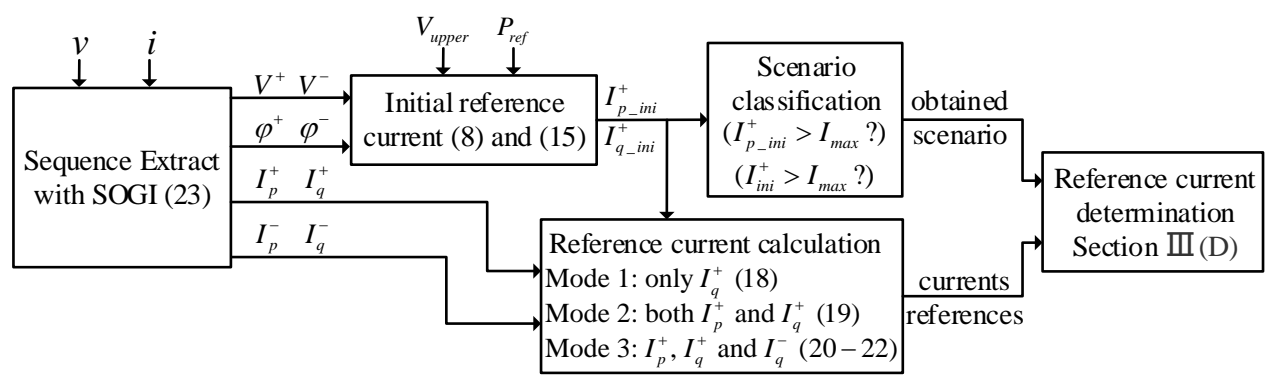

Figure 2. Control diagram of the proposed strategy

scenario matches with the related current injection modes as stated in section $\mathrm{C}$.

For serious voltage sag scenario $\left(I_{q_{-} \text {ini }}^{+}>I_{\max }\right)$, the reference current for $I_{q_{-}}^{+}$ref must be chosen as $I_{\max }$ to fully use the capacity without overcurrent, and none of the other current components (e.g. $I_{p}^{+}, I_{p}^{-}$and $I_{q}^{-}$) should be injected to the grid.

During unserious voltage sag and high level active power generation scenario $\left(I_{q_{-} i n i}^{+}<I_{\max }\right.$ and $\left.I_{i n i}^{+}>I_{\max }\right)$, the initial voltage support is achieved within the current limitation by injecting $I_{q_{-} i n i}^{+}$. Thus, the final reactive current reference $I_{q_{-} r e f}^{+}$can be determined as $I_{q_{-} i n i}^{+}$. And the large initial value of $I_{p_{-} \text {ini }}^{+}$should be modified based on (15) while both NS currents $I_{p_{-}}^{-}$ref and $I_{q_{-}}^{-}$ref remain as zero in this scenario.

Similarly, for unserious voltage sag and low level active power generation scenario $\left(I_{q_{-} i n i}^{+}<I_{\max }\right.$ and $\left.I_{\text {ini }}^{+}<I_{\max }\right)$, both initial voltage support and active power output requirements are satisfied. Thus, the reference currents for $I_{p_{-} r e f}^{+}$and $I_{q_{-} r e f}^{+}$are chosen as $I_{p_{-} i n i}^{+}$and $I_{q_{-} i n i}^{+}$respectively. To fully use the capacity of the inverter, the reference value for $I_{q_{-}}^{-}$ref is required and can be obtained by (16-18).

\section{Simulation Results}

In this section, three cases are tested to verify the proposed control strategy with three different scenarios. The simulations are performed based on MATLAB/SIMULINK, with a $15 \mathrm{kVA}$, $400 \mathrm{~V}$ IIDG connected to the grid side bus through a conductor $\left(X_{g}=\mathrm{j} 0.628 \Omega\right)$ as shown in Fig. 1 . The maximum current $I_{\max }$ is setting as 1.2 p.u. An unbalanced voltage sag happens at $\mathrm{t}=0.1 \mathrm{~s}$ and is been cleared at $\mathrm{t}=0.3 \mathrm{~s}$. Between $\mathrm{t}=0.2 \mathrm{~s}$ and $\mathrm{t}=0.3$ $\mathrm{s}$, the inverter operates with the proposed control strategy, while the PQ control is applied during other operation periods.

\section{A. Case A: Serious voltage sag (only inject $I_{q}^{+}$)}

This test aims to show the performance of the proposed control strategy with scenario 1 , where the initial active power $P_{r e f}$ is setting as 1 p.u., and a serious voltage sag happens at $\mathrm{t}=0.1 \mathrm{~s}\left(V^{+}=0.56\right.$ p.u. and $V^{-}=0.22$ p.u. $)$. Fig. 3(a) and (b) show that by activating proposed control strategy at $\mathrm{t}=0.2 \mathrm{~s}$, both the phase voltages and the PS voltage are supported $\left(V^{+}\right.$increased from 0.56 p.u. to 0.728 p.u.). However, due to the phase current constraints, the voltages in phase $\mathrm{b}$ and $\mathrm{c}$ remains below $V_{\text {upper }}=1.1$ p.u., which means the initial voltage support is

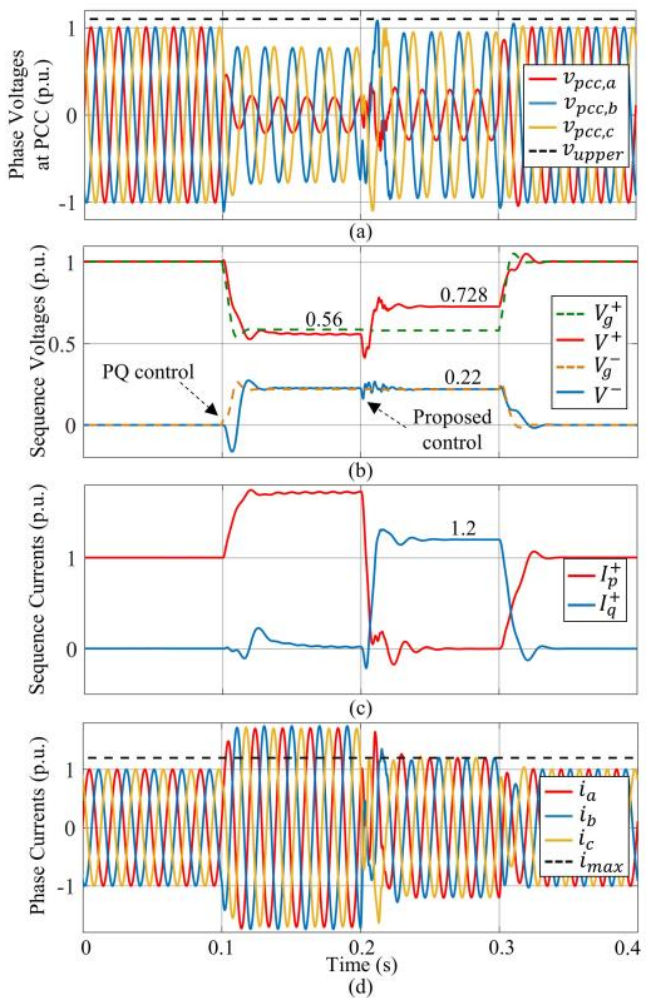

Figure 3. Simulation results for Case 1: (a) Phase voltages. (b) Sequence voltages. (c)Phase currents. (d) Sequence currents.

failed. As shown in Fig. 3(c) and (d), both PS current $I_{q}^{+}$and phase currents are well controlled within $I_{\max }=1.2$ p.u. Meanwhile, no active current is injected in this case.

\section{B. Case B: Unserious voltage sag and high active power generation(inject $I_{p}^{+}$and $I_{q}^{+}$)}

In this scenario, $P_{r e f}$ is setting as 1 p.u. and an unserious voltage sag appears at $\mathrm{t}=0.1 \mathrm{~s}\left(V^{+}=0.7\right.$ p.u. and $V^{-}=0.3$ p.u. $)$. As depicted in Fig. 4(a) and (b), the voltage support capability is fully enhanced, where $v_{c}$ is improved to 1.1 p.u., and $V^{+}$is increased to 0.81 p.u. Meanwhile, Fig. 4(c) shows that the active reference current calculated by equation (15) is required to be injected, which is 0.8 p.u. (lower than $I_{p_{-} i n i}^{+}=1.44$ p.u.). As shown in Fig. 4(d), the balanced three phase currents are reach to $I_{\max }$, and thus the capacity can be fully used. 

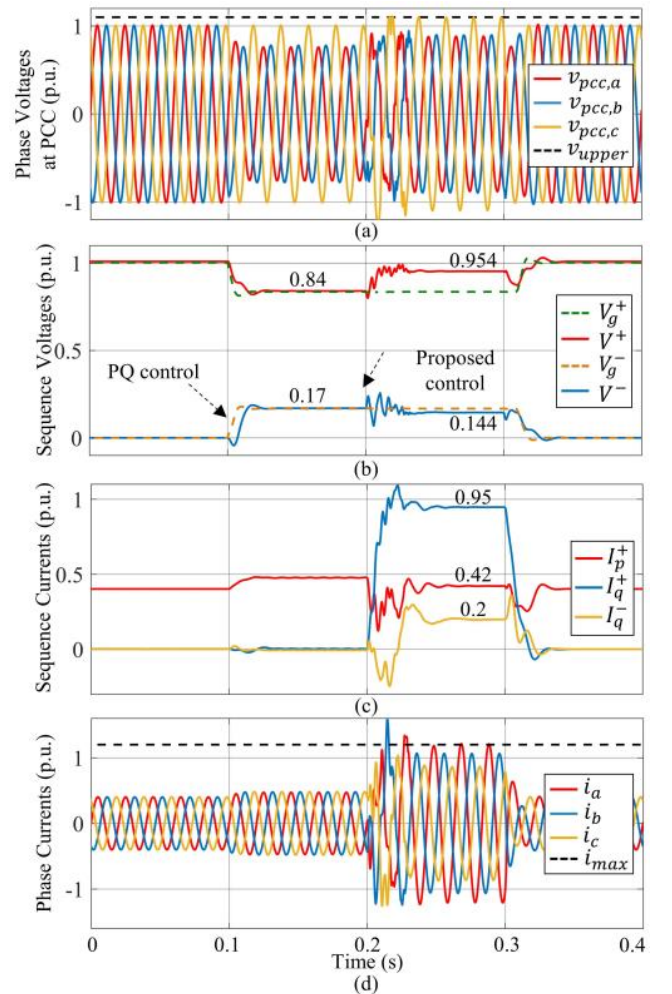

Figure 4. Simulation results for Case 2: (a) Phase voltages. (b) Sequence voltages. (c)Phase currents. (d) Sequence currents.

\section{Case C: Unserious voltage sag and low active power generation (inject $\mathrm{I}_{p}^{+}, I_{q}^{+}$and $I_{q}^{-}$)}

For this scenario, $P_{\text {ref }}$ is chosen as 0.4 p.u. (low level), and the sequence voltage $V^{+}$sag to 0.84 p.u. while $V^{-}$increase to 0.17 p.u.. As shown in Fig. 5(a) and (b), the initial voltage support requirement is satisfied, with $V^{+}$supported to 0.954 p.u. Furthermore, by the injection of the negative sequence reactive current upon (22), the NS voltage is decreased by 0.026 p.u.. As indicated in Fig. 5(d), though the three phase current remains unbalanced between $\mathrm{t}=0.2 \mathrm{~s}$ and $\mathrm{t}=0.3 \mathrm{~s}$, they are well controlled without overcurrent risk.

\section{CONCLUSION}

In this paper, a new fault control method is presented for IIDG with three control objectives, as to enhance its ride through capability within the related constraints. The main control objectives of the strategy are to maximize the PS voltage support capability and to ensure the phase current limits, while the active current injection is regarded as an ancillary service. Three operation scenarios are classified and implemented for the calculation of the reference currents. Simulation results have verified the effectiveness of the proposed fault control strategy under different scenarios.

\section{REFERENCES}

[1] Y. Yang, P. Enjeti, F. Blaabjerg and H. Wang, "Wide-Scale Adoption of Photovoltaic Energy: Grid Code Modifications Are Explored in the Distribution Grid," IEEE Industry Applications Magazine, vol. 21, no. 5, pp. 21-31, Sept.-Oct. 2015.
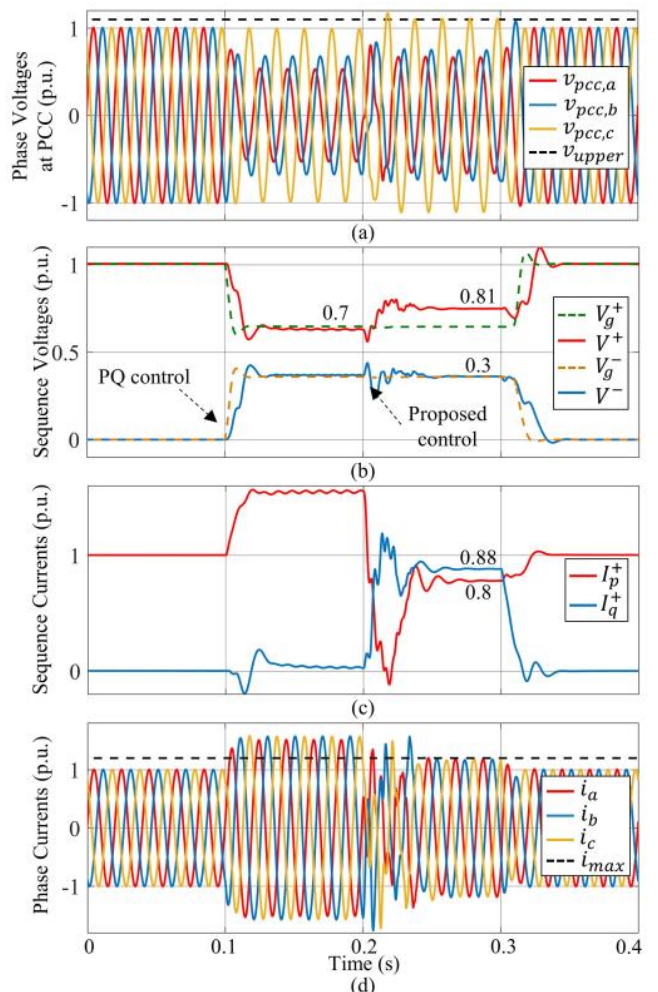

(d)

Figure 5. Simulation results for Case 3: (a) Phase voltages. (b) Sequence voltages. (c)Phase currents. (d) Sequence currents.

[2] M. M. Shabestary and Y. A. I. Mohamed, "Advanced Voltage Support and Active Power Flow Control in Grid-Connected Converters Under Unbalanced Conditions," IEEE Trans. on Power Electronics, vol. 33, no. 2, pp. 1855-1864, Feb. 2018.

[3] Grid Code for High and Extra High Voltage, E.ON Netz GmbH, Bayreuth, Germany, Apr. 2006.

[4] S. Alepuz et al., "Control Strategies Based on Symmetrical Components for Grid-Connected Converters Under Voltage Dips," IEEE Trans. on Industrial Electronics, vol. 56, no. 6, pp. 2162-2173, June 2009.

[5] F. Wang, J. L. Duarte and M. A. M. Hendrix, "Pliant Active and Reactive Power Control for Grid-Interactive Converters Under Unbalanced Voltage Dips," IEEE Trans. on Power Electronics, vol. 26, no. 5, pp. 1511-1521, May 2011.

[6] P. Piya, M. Ebrahimi, M. Karimi-Ghartemani and S. A. Khajehoddin, "Fault Ride-Through Capability of Voltage-Controlled Inverters," IEEE Trans. on Industrial Electronics, vol. 65, no. 10, pp. 7933-7943, Oct. 2018.

[7] Z. Shuai, C. Shen, X. Yin, X. Liu and Z. J. Shen, "Fault Analysis of Inverter-Interfaced Distributed Generators With Different Control Schemes," IEEE Trans.on Power Delivery, vol. 33, no. 3, pp. 12231235, June 2018.

[8] A. Camacho, M. Castilla, J. Miret, P. Martí and M. Velasco, "Maximizing positive sequence voltage support in inductive-resistive grids for distributed generation inverters during voltage sags," IECON 2016-42nd Annual Conference of the IEEE Industrial Electronics Society, Florence, 2016, pp. 2343-2348.

[9] W. Guo, L. Mu and X. Zhang, "Fault Models of Inverter-Interfaced Distributed Generators Within a Low-Voltage Microgrid," IEEE Trans. on Power Delivery, vol. 32, no. 1, pp. 453-461, Feb. 2017.

[10] A. Camacho, M. Castilla, J. Miret, R. Guzman and A. Borrell, "Reactive Power Control for Distributed Generation Power Plants to Comply With Voltage Limits During Grid Faults," IEEE Trans. on Power Electronics, vol. 29, no. 11, pp. 6224-6234, Nov. 2014.

[11] M. M. Shabestary and Y. A. I. Mohamed, "Asymmetrical Ride-Through and Grid Support in Converter-Interfaced DG Units Under Unbalanced Conditions," IEEE Trans. on Industrial Electronics, vol. 66, no. 2, pp. 1130-1141, Feb. 2019. 